\title{
Saccbaromyces telluris, \\ une levure de la flore digestive animale, particulièrement fréquente chez le Babouin, Papio papio
}

\author{
par H. SAEZ, J.-P. HUGOT et F. TRAORE \\ Laboratoire d'Ethologie du Muséum national d'Histoire naturelle, \\ Parc zoologique, 53, avenue de Saint-Maurice, F 75012 Paris
}

\section{Résumé.}

Le Saccharomyces telluris Van der Walt apparaît comme une levure relativement peu commune de la flore digestive des animaux de la collection du Parc zoologique de Paris. Dans une investigation portant sur quelque 14 années, il a été isolé chez 39 des 2110 sujets autopsiés. Il est présent chez $0,9 \%$ des Mammifères (et l'hôte de 11 espèces animales) et $0,8 \%$ des Oiseaux (et l'hôte de 6 espèces aviaires). En revanche, il est hébergé par $22,8 \%$ des 92 Babouins - Papio papio (Desm.) - examinés au cours de cette période ; le taux s'élève à $27,1 \%$ si, retenant les résultats de la forme imparfaite, on considère le couple $S$. telluris-Torulopsis bovina. La fréquence de $S$. telluris chez le Babouin est proche de celle de Candida albicans et bien supérieure à celle des autres constituants de la flore levuriforme. On le rencontre plus communément chez les sujets mâles, ainsi que chez les sujets les plus âgés, toujours à l'état non parasitaire. Cette levure thermotolérante (température maximale de développement: $43^{\circ}-46^{\circ}$ ) peut prospérer à tous les étages du tube digestif.

\section{Summary.}

Saccharomyces telluris, a yeast of the animal digestive flora, particularly common in the Baboon, Papio papio.

Saccharomyces telluris Van der Walt is a yeast not very common for the whole of the animals of the Paris's zoological Park in their digestive tract: 39 strains isolated on 
2110 subjects. This fungus occurs in $0,9 \%$ of the Mammals (II animal species-host) and in $0,8 \%$ of the Birds ( 6 avian species-host). But in the Baboon-Papio papio (Desm.), the percentage increases to $22,8 \%$ and even to $27,1 \%$ if we consider the couple perfect and inperfect form: Saccharomyces telluris-Torulopsis bovina. For the Baboon, the frequence of $S$. telluris is near that of Candida albicans and greater than that of other yeastflora components. Always at a non-parasitic state, this yeast is more common in the males and in the oldest subjects. S. telluris, a thermotolerante yeast (maximal for growth : $43^{\circ}-46^{\circ}$ ) can prosper at every stages of the digestive tract.

Les levures de la flore du tube digestif de l'homme, des mammifères et des oiseaux se distinguent en plusieurs catégories selon le critère retenu. La spécificité, plus ou moins étroite, à un hôte donné intéresse l'écologiste, sans laisser indifférent le mycopathologiste.

En fonction de l'habitat on peut séparer les levures en trois groupes: Groupe I, levures qui se multiplient dans la nature et sont aptes à le faire aussi, éventuellement, chez l'homme et l'animal. Groupe 2, levures qui sont plus spécialement adaptées à vivre dans un organisme à sang chaud et hébergées par beaucoup d'espèces animales. Groupe 3, les levures, parmi celles du Groupe 2, que l'on ne rencontre que chez un petit nombre d'hôtes.

Des trois groupes ci-dessus considérés, le Groupe I est celui qui subit le plus fortement l'influence du rythme saisonnier (4). Les thermotolérances les plus élevées sont surtout l'apanage des levures des Groupes 2 et 3. Quant aux exigences culturelles les plus grandes (besoins vitaminiques, thermiques), on les rencontre, le plus communément, chez les levures du Groupe 3.

D'une façon plus générale, l'âge (3) et le régime alimentaire (7) ont une grande incidence sur la flore fongique: la flore digestive la plus riche, la plus diversifiée, se voit chez les omnivores; chez les carnivores et les piscivores, les levures sont rares.

Candida albicans est l'espèce la plus représentative du Groupe 2. Il est hébergé par l'homme et maints mammifères et oiseaux; sa fréquence, l'activité de son bourgeonnement, voire l'exaltation de son pouvoir pathogène et son passage au parasitisme varient sous l'influence de certains facteurs: l'âge et l'état physio-pathologique du sujet, son régime alimentaire, l'administration de produits médicamenteux; chez le même individu on le rencontre à tous les étages du tube digestif ou à quelques niveaux seulement de celui-ci. A l'opposé, Saccharomycopsis guttulata n'est pratiquement observé que chez le Lapin (et des espèces voisines sauvages); pendant longtemps les essais de culture de cette levure du Groupe 3 sont restés infructueux.

Entre ces deux cas extrêmes, il existe bien des degrés dans l'adaptation à l'hôte et la colonisation du tube digestif. De plus, une levure qui paraissait, à l'origine, particulièrement bien adaptée à un organisme se révèle, dans des investigations ultérieures, capable de prospérer dans d'autres organismes. Actuellement on peut encore 
ranger dans le Groupe 3 : Candida slooffii (hôtes : Cheval, Porc) (2), (10); Torulopsis (Candida) bovina (hôtes: Cheval, Porc, Dindon) (2), (9); Torulopsis pintolopesii (hôtes: Souris blanche, Rat, Chinchilla, Pigeon) (2), (10), (11). Nous plaçons également dans le Groupe 3 Trichosporon capitatum: ainsi que les trois espèces citées, il a une température maximale élevée $\left(45^{\circ}-47^{\circ}\right.$ d'après nos souches) ; en revanche, il se développe aisément et sur les milieux de culture habituels et à la température du laboratoire. $T$. capitatum, trouvé dans la nature, a par la suite été identifié chez l'homme et quelques espèces animales; dans notre population, nous le rencontrons presque uniquement chez les Suidés sauvages (Potamochère, Phacochère), comme constituant de la flore digestive et jamais, jusqu'à présent, à l'état parasitaire (8).

Les résultats que nous rapportons ici concernent le Saccharomyces (tellustris) telluris (2), (12). Il s'agit d'une levure thermotolérante, à croissance lente et faible surtout à la température du laboratoire. Une étude comparée a conduit Kreger-Van Rij à considérer $S$. telluris comme la forme parfaite de $T$. bovina (1). Van der Walt l'a isolé, pour la première fois, du sol; il apparaît néanmoins comme un constituant de la flore fongique de certains homéothermes. Nous avons déjà enregistré sa présence dans l'appareil digestif de plusieurs mammifères et oiseaux sauvages; sa diffusion chez l'animal demeure, cependant, bien moins large que celle de $C$. albicans et sa place serait encore, à notre avis, parmi les levures du Groupe 3. La distribution de S. telluris est analysée chez 2110 sujets de la Collection du Parc zoologique de Paris observés en 14 ans et, plus particulièrement, chez le Babouin, Papio papio (Desm.).

\section{Matériel et méthodes}

De janvier 1959 à juillet 1973, nous avons mené nos investigations sur les animaux sauvages morts en captivité au Parc zoologique de Paris. Au cours de l'autopsie des prélèvements sont pratiqués, d'une part, au niveau de lésions mycosiques quand elles existent, d'autre part, systématiquement, en plusieurs endroits de l'organisme animal. Seuls sont retenus, dans cette analyse, les résultats concernant les prélèvements du tube digestif.

Dans le tube digestif le matériel est prélevé :

- Chez les mammifères, au niveau : du pharynx, de l'intestin grêle, du gros intestin, du rectum et, uniquement chez les sujets nouveau-nés âgés au plus de 10 jours, de l'estomac.

- Chez les oiseaux, au niveau: du pharynx, de l'intestin, de l'un des caeca, si le sujet en possède, et du rectum.

Le matériel pour cultures est dilué dans $5 \mathrm{cc}$ d'eau peptonée à $1 \%$, avec lesquels nous ensemençons deux boîtes de Petri, contenant du Sabouraud glucosé à $2 \%$; l'une des boîtes est mise dans une étuve à $37^{\circ}$ et l'autre laissée à la température du laboratoire. 
L'étude enzymologique est faite à $25^{\circ}$; les galeries d'identification sont conservées, en général, au plus un mois à l'étuve. Pour déceler une fermentation, nous utilisons la technique de Guerra: eau peptonée + produit à tester, en tubes droits de $14 \times 140$, bouchon de paraffine coulé à la surface du milieu liquide. Pour l'auxanographie nous employons le milieu gélosé : milieu classique pour assimilation, vitaminé + produit à tester, en tubes de $16 \times 160$. La température maximale de développement est recherchée en tubes de $16 \times 160$, sur milieu de Sabouraud glucosé $2 \%$ : la limite thermique supérieure est supposée atteinte quand aucune croissance n'est notée après 15 jours d'exposition à une température donnée.

\section{Résultats}

\section{I. - Résultats des prélèvements.}

\begin{tabular}{|c|c|c|}
\hline & $\begin{array}{c}\text { Nombre de sujets } \\
\text { examinés }\end{array}$ & $\begin{array}{c}\text { Nombre de sujets } \\
\text { hébergeant } \\
S . \text { telluris }\end{array}$ \\
\hline Total des animaux $\ldots \ldots \ldots \ldots \ldots$ & 2110 & 39 \\
\hline Dont : - oiseaux $\ldots \ldots \ldots \ldots \ldots$ & 890 & 7 ou $0,8 \%$ \\
\hline - mammifères $\quad \ldots \ldots \ldots \ldots \ldots$ & 1220 & 32 ou $2,5 \%$ \\
\hline $\begin{array}{c}\text { mammifères sans les ba- } \\
\text { bouins } \ldots \ldots \ldots \ldots \ldots \ldots \ldots\end{array}$ & 1128 & 11 ou $0,9 \%$ \\
\hline - Babouins $\ldots \ldots \ldots \ldots \ldots \ldots$ & 92 & 21 ou $22,8 \%$ \\
\hline
\end{tabular}

$S$. telluris a été identifié 61 fois, à savoir :

- 17 fois dans la cavité buccale;

- 17 fois dans l'intestin grêle;

- 16 fois dans le rectum;

- 8 fois dans le gros intestin (mammifères);

- 2 fois dans l'un des caeca (oiseaux);

- 1 fois dans l'estomac (mammifères nouveau-nés).

$\mathrm{Au}$ total, nous avons enregistré 39 sujets-hôtes appartenant à 18 espèces animales.

A) Oiseaux. S. telluris a été décelé chez:

- 1 Paon bleu, Pavo cristatus ................... sur 17 sujets examinés

- 1 Cigogne blanche, Ciconia ciconia .............. > 15 , 》

- 1 Flamant de James, Phoenicoparrus jamesi ........ \. 10 》 \$

- 1 Néophron percnoptère, Neophron percnopterus ..... \3 3 》

- 2 Dindons sauvages, Meleagris gallopavo ........... 》 2 》

-1 Canard pilet du Chili, Anas georgica spinicanda .... \ 1 » 》 
Pour ces 7 sujets-hôtes, appartenant à 6 espèces aviaires, S. telluris s'est multiplié :

- 4 fois dans un étage seulement du tube digestif;

- 3 fois dans deux étages seulement du tube digestif;

- jamais dans tout le tube digestif, ni dans trois des quatre étages observés chez les oiseaux.

B) Mammifères sans les Babouins. S. telluris a été isolé chez:

-1 Cerf d'Eld, Rucervus eldi .................. sur 52 sujets examinés

- 1 Antilope indienne, Antilope cervicapra ........... \43 》 \$

-1 Gazelle dorcas, Gazella dorcas ............... , 17 , 》

-1 Renne, Rangifer tarandus .................. \$ 14 , >

-1 Cerf des marais, Hydropotes inermis ........... , 11 , >

-1 Gazelle de Perse, Gazella subgutturosa .......... \ 7 » 》

- 1 Panthère, Panthera pardus .................. \$ 5 ,

-1 Ragondin, Myopotamus coypus .............. > 4 》 》

- 1 Castor d'Europe, Castor fiber ................ 》 4 》

- 1 Céphalophe à dos jaune, Cephalophus sylvicultor ... \ 1 》 ,

-1 Caracal, Felis caracal ..................... \$ 1 ,

La colonisation de ces 11 hôtes s'est faite ainsi :

- 6 fois dans un étage seulement du tube digestif ;

-2 fois dans deux étages;

-2 fois dans trois étages;

-1 fois dans tout le tube digestif.

C) Babouins. Pendant la période sous revue, 174 Primates ont été autopsiés ; S. telluris n'a été identifié que sur les Babouins : 21 ou $22,8 \%$ de sujets-hôtes sur 92 .

En fonction du sexe, la répartition se fait ainsi :

— Babouins mâles : 40 sujets examinés, 11 positifs ou $27,5 \%$;

- Babouins femelles: $52, \quad, 10 \gg, 19,2 \%$.

Trois Babouins sont des mort-nés; deux d'entre eux étaient déjà porteurs de $S$. telluris (prélèvement pharyngé positif). Pour les 89 sujets nés vivants, l'influence de l'âge sur la fréquence de ce Saccharomyces est la suivante:

— sujets âgés au plus de 10 jours : 1 positif sur 14 ou $7,1 \%$;

— sujets âgés de plus de 10 jours à 1 an : 6 positifs sur 36 ou $16,6 \%$;

— sujets âgés de plus d'un an: 12 positifs sur 39 ou $30,7 \%$.

La forme imparfaite, $T$. bovina, a été trouvée chez 4 Babouins (n'hébergeant pas S. telluris) :

— un sujet mâle, âgé de 3 jours, dans la cavité buccale ;

— un sujet mâle, âgé de 10 jours, dans la cavité buccale et le gros intestin ;

- un sujet mâle, âgé de 4 mois $1 / 2$, dans l'intestin grêle; 
— un sujet femelle, âgé de 5 mois $1 / 2$, dans la cavité buccale, l'intestin grêle, le gros intestin et le rectum.

Compte tenu de ces derniers résultats, ce sont 25 ou $27,1 \%$ des Babouins qui hébergent le couple $S$. telluris-T. bovina.

\section{II. - Le Saccharomyces telluris.}

a) Chez l'animal. Deux fois nous avons surpris, à l'examen microscopique direct, une multiplication active de $S$. telluris, à l'état non parasitaire, dans la cavité buccale. Les blastospores sont grandes (néanmoins plus petites que celles de la même souche cultivée sur Sabouraud glucosé à $2 \%$ ), ovales, en poire ou arrondies; le bourgeon se détache tôt de la cellule-mère. Aucune formation pseudomycélienne n'a été notée.

b) En culture.

- En primo-culture, sur Sabouraud glucosé à $2 \%$, les colonies apparaissent rapidement dans la boîte de Petri incubée à $37^{\circ}$ : en 24 heures, plus communément en 2 à 3 jours. Elles sont petites : 1 à $2 \mathrm{~mm}$ de diamètre, dépassant rarement $3 \mathrm{~mm}$ même dans une primo-culture âgée. Ces colonies sont brillantes, lisses et de couleur beige clair pendant les tout premiers jours, puis se ternissent et deviennent beige foncé.

- En culture, sur Sabouraud glucosé à $2 \%$, le diamètre de la colonie est toujours plus grand qu'en primo-culture et la couleur varie du blanc ou de l'ivoire au beige foncé. Les cellules, ovales, ovoïdes ou rondes, mesurent $(4,5-7,5)(5-10,5) \mu$. Sur Maltea-Moser à $1 \%$ l'absence totale de filamentisation, tout comme une filamentisation abondante, est rare; le plus généralement il se forme un pseudomycélium rudimentaire, des ébauches ou des tubes mycéliens. La sporulation est plus ou moins importante: jusqu'ici nous n'avons vu qu'une seule ascospore par asque, ascospore ronde ou légèrement ovale, à paroi lisse.

- Fermentation : des sept produits testés (glucose, galactose, saccharose, maltose, lactose, raffinose et inuline), seul le glucose est fermenté : faiblement en général, vigoureusement par certaines souches.

- Assimilation :

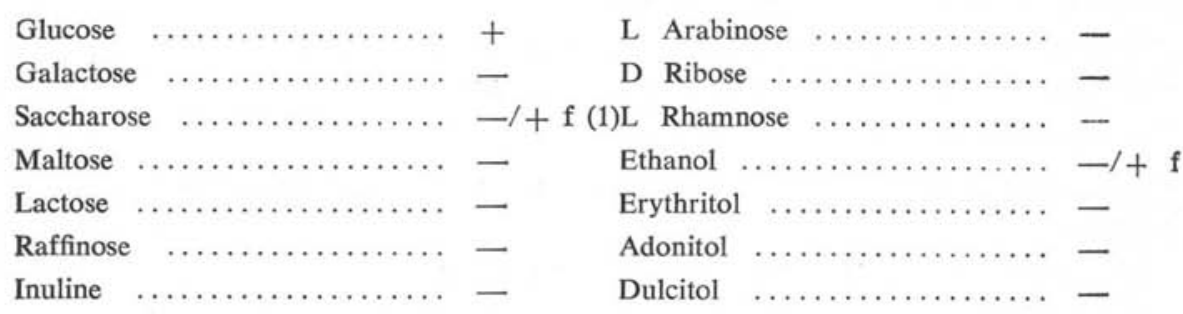

(1) 2 souches seulement ont donné une réaction faiblement positive avec le saccharose. 


\begin{tabular}{|c|c|c|c|}
\hline Cellobiose & $\ldots \ldots \ldots \ldots \ldots \ldots$ & 一 & D Mannitol $\ldots \ldots \ldots \ldots \ldots \ldots$ \\
\hline Tréhalose & $\ldots \ldots \ldots \ldots \ldots \ldots \ldots$ & - & D Sorbitol $\ldots \ldots \ldots \ldots \ldots \ldots$ \\
\hline D Xylose & $\ldots \ldots \ldots \ldots \ldots \ldots$ & - & Inositol $\ldots \ldots \ldots \ldots \ldots \ldots \ldots$ \\
\hline
\end{tabular}

\section{Discussion}

Parmi les levures de la flore digestive, le $S$. telluris est une espèce dont on peut suspecter la présence: in vivo, par la taille, plus encore que par la forme, de ses cellules qui sont plus grandes que celles des autres espèces observées le plus communément et, en particulier, plus grandes que celles de $C$. albicans; in vitro, par le développement assez rapide (en 24 à 72 heures), en primo-culture à $37^{\circ}$ sur Sabouraud glucosé $2 \%$, de petites colonies en tête d'épingle de couleur beige clair à beige foncé. Et à propos de la taille des éléments fongiques, nous avons remarqué que les levures ont, en général, des blastospores légèrement plus petites in vivo (examen microscopique direct de matériel du tube digestif dans de l'eau physiologique) qu'en culture pure sur Sabouraud glucosé $2 \%$; eu égard aux variations sensibles des dimensions cellulaires in vitro, les deux précisions: culture pure et sur Sabouraud glucosé ont leur importance (l'examen microscopique d'une culture ayant lieu, également, dans une goutte d'eau physiologique).

Si l'on s'arrête aux résultats globaux (39 sujets positifs sur 2110 ), le $S$. telluris apparaît comme un constituant plutôt rare de la flore levuriforme des animaux sauvages captifs. Et si l'on retranche les résultats relatifs au Babouin, on constate que la fréquence de cette levure est faible et du même ordre de grandeur chez les mammifères et les oiseaux : respectivement 0,9 et $0,8 \%$. Enfin, quand on compare les résultats des diverses espèces animales, pour lesquelles un échantillon de sujets assez important permet une confrontation valable, on est frappé par le bond du taux de positivité chez le Babouin : 22,8\%.

Le rôle de $S$. telluris dans la flore digestive du Babouin prend davantage de relief lorsque l'on rapproche ses résultats de ceux des autres levures thermiquement aptes à se multiplier chez l'animal identifiées au cours de cette investigation, à savoir :

- Candida albicans: température maximale $42^{\circ}-47^{\circ}$;

- C. krusei : température maximale $44^{\circ}-47^{\circ}$;

- C. tropicalis: température maximale $42^{\circ}-46^{\circ}$;

- C. guilliermondii : température maximale $39^{\circ}-43^{\circ}$;

- C. parapsilosis: température maximale $38^{\circ}-44^{\circ}$;

- Torulopsis glabrata: température maximale $44^{\circ}-46^{\circ}$. 
Au niveau d'un ou plusieurs étages du tube digestif du Babouin, nous avons décelé le développement à l'état non parasitaire (et à l'état parasitaire en ce qui concerne $C$. albicans : à l'origine d'une candidose buccale chez 6 sujets) de :

- C. albicans ..................... chez 25 des 92 babouins examinés.



- C. tropicalis ..................... 》 4 》 》

- C. guilliermondii ................... 》 3 » 》 》

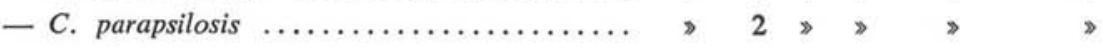

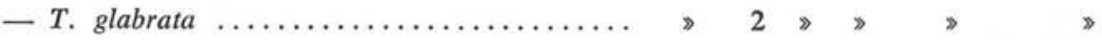

Ainsi donc, les deux principaux constituants de la flore digestive du Babouin sont le $C$. albicans et le $S$. telluris : le premier est un peu plus souvent rencontré que le second, mais, si l'on retient les résultats de la forme imparfaite, le couple $S$. telluris$T$. bovina est aussi fréquent que le $C$. albicans. Les autres espèces fongiques sont bien moins communément hébergées.

Dans notre échantillon, le Babouin mâle est plus fréquemment l'hôte de S. telluris que le Babouin femelle (27,5\% contre 19,2 \%). En fonction de l'âge nous avons obtenu : quelque 4 fois plus de sujets positifs chez les sujets âgés de plus d'un an que chez les nouveau-nés. Mais nous avions déjà noté que la fréquence, voire la diversité, de la flore levuriforme des mammifères augmente généralement en fonction de l'âge des animaux (3). L'isolement de ce Saccharomyces de la cavité buccale de deux sujets mort-nés montre que la transmission, sinon le développement, a lieu très tôt, pendant le passage parfois.

En définitive, dans notre population d'animaux sauvages captifs, le $S$. telluris est une levure relativement peu commune, capable de se développer, occasionnellement, passagèrement peut-être, chez quelques espèces de mammifères et d'oiseaux. Il apparaît en revanche comme un micromycète bien adapté au tube digestif du Babouin, chez lequel on le rencontre dans près d'un sujet sur quatre, et où il peut prospérer à tous les étages du tube digestif.

\section{Bibliographie}

1. Kreger-VAN RiJ (N.) (J. W.), 1958. - The relationship between Saccharomyces tellustris and Candida bovina. Ant. Leeuwenh., 24, 137-144.

2. Lodder (J.), 1971. - The Yeasts. A taxonomic study. $2^{\circ}$ éd., North-Holland Cy, Publ., Amsterdam.

3. SAËZ (H.), 1969. - Levures isolées du tube digestif des mammifères examinés de 1959 à 1963. Résultats en fonction de l'âge. Ann. Inst. Past., 116 (2), 218-236.

4. —, 1969. - L'incidence saisonnière sur les levures isolées chez des animaux sauvages captifs. Bull. Sté Mycol. France, 85 (2), 255-271.

5. —, 1970. - Flore levuriforme intestinale du Babouin, Papio papio (Desm.). Mycopath. Mycol. Appl., 41, 383-395. 
6. - 1970. - Levures de la cavité buccale du Babouin, Papio papio (Desm.). Zbl. Vet. Med., B, 17, 381-388.

7. - et Rinjard (J.), 1970. - Levures isolées du tube digestif de Mammifères adultes. Résultats en fonction du régime alimentaire. Les Cahiers Méd. Vét., 39 (6), 290-304.

8. - et - 1973. - Trichosporon capitatum, un constituant de la flore fongique du tube digestif de certains Suidés. Ann. Méd. Vét., 117, 177-182.

9. Uden N. VAN et Do Carmo Sousa (L.), 1957. - Yeasts from the bovine caecum. J. Gen. Microbiol., 16, 385-395.

10. - et -, 1957. - Candida slooffii nov. sp., a thermophilic and vitamin deficient yeast from the equine intestinal tract. Portug. Acta Biol., A, 5, 7-17.

11. - , - et FARINHA (M.), 1958. - On the intestinal yeast flora of horses, sheep, goats and swine. J. Gen. Microbiol., 19, 435-445.

12. WALt (J.-P.) VAN DER, 1957. - Three new sporogenous yeasts from soil. Ant. Leeuwenh., 23, 23. 